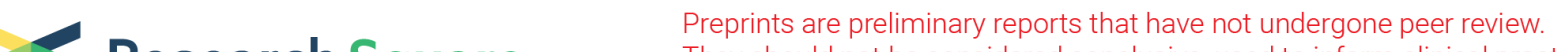 $\begin{array}{ll}\text { Research Square } & \text { They should not be considered conclusive, used to inform clinical practice, } \\ \text { or referenced by the media as validated information. }\end{array}$
}

\section{Global Burden of Non-communicable Diseases Attributable to Dietary Risks in 1990-2019}

Jie Qiao

Zhejiang University School of Medicine Second Affiliated Hospital

Xiling Lin

Zhejiang University School of Medicine Second Affiliated Hospital

Yiwen Wu

Ningbo City First Hospital

Xin Huang

Zhejiang University School of Medicine Second Affiliated Hospital

Xiaowen Pan

Zhejiang University School of Medicine Second Affiliated Hospital

Jingya Xu

Zhejiang University School of Medicine Second Affiliated Hospital

JunYun Wu

Zhejiang University School of Medicine Second Affiliated Hospital

Yuezhong Ren ( $\nabla$ renyuez@zju.edu.cn)

Department of Endocrinology and Metabolism, the Second Affiliated Hospital of ZheJiang University

School of Medicine, 88 Jiefang Road, Hangzhou, Zhejiang, 310009, China https://orcid.org/0000-00032732-8025

\section{Peng-Fei Shan}

Zhejiang University School of Medicine Second Affiliated Hospital

\section{Research}

Keywords: Dietary risks, Mortality, Disability-adjusted life-years, DALYs, Non-communicable diseases, Global Burden of Disease Study

Posted Date: January 5th, 2021

DOI: https://doi.org/10.21203/rs.3.rs-136723/v1

License: (c) (1) This work is licensed under a Creative Commons Attribution 4.0 International License. Read Full License 
Version of Record: A version of this preprint was published at Journal of Human Nutrition and Dietetics on June 23rd, 2021. See the published version at https://doi.org/10.1111/jhn.12904. 


\section{Abstract}

Background: Dietary risks raised attention around worldwide during the past decades. The aims of this burden-of-disease study were to evaluate the global dietary risks for non-communicable diseases (NCDs) from 1990 to 2019 and quantify their impact on mortality and disability-adjusted life-years (DALYs).

Method: Data from the 2019 Global Burden of Disease Study on deaths and DALYs from NCDs attributable to worldwide dietary risks were obtained and underwent deep analysis by year, age, gender, location, leading risks, and leading causes, and their associations were examined. The Sociodemographic Index (SDI) was used as an indicator of national socioeconomic status, and the relationships between age-standardized rates of deaths or DALYs and socioeconomic status.

Results: In 2019, 7.9 million deaths and 187.7 million DALYs were attributable to dietary risk factors. High intake of sodium and low intake of whole grains and fruits were leading dietary risks for deaths and DALYs worldwide. However, both indexes showed a decreasing trend by year, an increase by age, and a higher disease burden in males. The main distribution of dietary-related NCDs was located in highly populated countries. A negative association between the SDI and disease burden, and a positive association between the SDI and male preponderance were found.

Conclusions: Dietary risk factors for NCDs increased significantly and varied across regions during 19902019. Therefore, greater efforts are needed to raise public awareness of interventions and improve dietary practices to reduce the disease burden caused by suboptimal dietary intake, especially in developing countries and among males.

\section{Background}

Dietary risks are thought of as low nutritional intake and poor dietary habits; however, they primarily include high sodium intake and low intake of whole grains and fruits. In recent decades, death and disability-adjusted life-years (DALYs) attributable to suboptimal diets have increased significantly ${ }^{1}$ As a comprehensive measurement of diseases, DALYs quantifies health loss due to specific diseases and injuries ${ }^{2}$. Non-communicable diseases (NCDs) accounted for the greatest proportion of total deaths (74.4\%) in 2019, and its causes increased from 2009 to 2019 by $20.5 \%$, representing 7.1 million additional deaths in 2019 versus $2009^{3}$. NCDs (e.g., ischemic heart disease, stroke, diabetes mellitus, colon and rectal cancer, and hypertensive heart disease) are leading causes of global deaths and disability. They are common among persons of working age; thus, expenditures for NCDs exceed those for treating other diseases. The high burden of NCDs has raised concerns about the financial burden of follow-up, particularly, in low- and middle-income countries ${ }^{4}$.

This study examined the relationship between suboptimal dietary intake and NCDs with sociodemographic characteristics and year to evaluate the hazard more precisely. We aimed to assist in the effort to reduce the burden of NCDs and promote the implementation of the World Health 
Organization's Global Action Plan for the Prevention of NCDs 2013-2020 5 . The main foci of this action plan are four NCDs: cardiovascular disease, cancer, chronic respiratory disease, and diabetes, which contribute most to the morbidity and mortality of NCDs, and four behavioral risk factors, including tobacco use, unhealthy diet, physical inactivity, and harmful use of alcohol.

\section{Materials And Methods}

\section{Data source}

Suboptimal nutrition was found to be a risk factor for the development of NCDs in the 2019 Global Burden of Disease (GBD) Study ${ }^{6}$. We quantified the burden of the NCDs attributable to dietary risks from 1990 to 2019. Data for the analyses obtained from the Global Health Data Exchange consisted of (1) the numbers of males and females and age-standardized rates (per 100,000 population) of deaths and DALYs, annually from 1990 to 2019; (2) absolute numbers and unadjusted rates of global gender-agespecific deaths and DALYs in 2019; (3) countries' and regions' deaths and DALYs numbers and agestandardized rates in 2019; (4) global deaths and DALYs, including the number and age-standardized rate of each NCD attributable to dietary risks in 1990, 2010, and 2019; (5) global deaths and DALYs, including the number and age-standardized rate of each dietary risk, which led to NCDs in 1990, 2010, and 2019; and (6) age-standardized rates of deaths and DALYs in the GBD super regions in 1990, 2010, and 2019. Ethics approval and informed consent were not required for this study, as the data were accessible to the public.

Socioeconomic status was measured using the Socio-demographic Index (SDI), "a composite indicator of development status strongly correlated with health outcomes. It is the geometric mean of 0 to 1 indices of total fertility rate under the age of 25 , mean education for those ages 15 and older, and lag distributed income per capita. As a composite, a location with an SDI of 0 would have a theoretical minimum level of development relevant to health, while a location with an SDI of 1 would have a theoretical maximum level" ${ }^{20}$. The SDI of 195 countries and territories in the 2019 GBD study were divided into five categories: high SDI (>0.81), high-middle SDI (0.70-0.81), middle SDI (0.61-0.69), low-middle SDI (0.46-0.60), and low SDI $(<0.46)$.

\section{Statistical analysis}

Analyzed data are expressed as a value with a 95\% uncertainty interval (UI). Age-standardized rates of death and DALYs are expressed as a number per 100,000 population. The association of agestandardized rates of deaths and DALYs with SDI were analyzed using linear regression. All statistical analyses (unless otherwise specified) were conducted with GraphPad Prism (version 8). A P value less than 0.05 was considered statistically significant.

\section{Results}




\section{Global trends in NCDs attributable to dietary risks by year}

Globally, 42.0 million (95\%Ul, 40.0-43.9) deaths were attributed to NCDs in 2019; among them, 7.9 million (95\%Ul, 6.5-9.8) accounted for $28.5 \%$ of all risk-related NCDs attributable to dietary risks, which had risen from 5.4 million (95\%UI, 4.5-6.5) in 1990. The DALYs reached 187.7 million (95\%UI, 156.0-255.4) in 2019, accounting for $25.2 \%$ of all DALYs of NCDs, which had increased from 132.0 million (95\%Ul, 111.3-156.8) in 1990 (Fig. 1).

The death rate, which was standardized by age and population, decreased from 154.3 (95\%UI, 113.3166.3 ) in 1990 to 101.0 (95\%UI, 82.0 to 124.3 ) per 100,000 population in 2019. Males showed a similar trend to females, but higher rates of death and DALYs, from 1990 to 2019. In 2019, the mortality was 126 (95\%Ul, 102.7-154.5) among males and 80.0 (95\%Ul, 63.5-99.7) among females, and the DALY rate was 2911.5 (95\%UI, 2402.2-3495.1) in males and 1709.6 (95\%Ul, 1395.6-2091.6) in females.

\section{Global trends in NCDs attributable to dietary risks by gender, and age}

Global gender differences showed more severe consequences (i.e., higher death and DALY rates) in males from 1990 to 2019 (Fig. 1). In 2019, 3.5 million (95\%Ul, 2.8-4.4) deaths were reported in women and 4.5 million ( $95 \% \mathrm{UI}, 3.6-5.4)$ in men; the death rate per 100,000 population was 80.0 (95\%Ul, 63.5-99.7) in women and 126 (95\%UI, 102.7-154.5) in men; the DALYs were 74.3 million (95\%UI, 60.6-91.0) in women and 113.4 million (95\%Ul, 93.4-135.6) in men; and the DALY rate was $1710.0(95 \% \mathrm{UI}, 1396.1-2092.0)$ in women and $2911.5(95 \% \mathrm{UI}, 2402.2-3495.1)$ in men.

Trends in the numbers of deaths and DALYs attributable to dietary risks in both genders changed substantially with age (Fig. 2), with males peaking at age 70-74 with 567603.0 (95\%Ul, 449339.8709158.629.1) deaths and females peaking at age 80-84, with 545559.2 (95\%Ul, 428615.7-697221.4) deaths. The number of DALYs peaked for both genders at age $65-69$ with 23.9 million (95\%UI, 19.329.3), among females of a similar age at 9.3 million (95\%UI, 7.4-11.6), and among males age 6569 years at 15.0 million (95\%UI, 12.118.2).

Before age 80, males had more deaths and DALYs, while females appeared to exceed those numbers after age 80; however, males in all age groups always had higher death and DALY rates. Deaths among those under 80 years accounted for $69.8 \%$ of all deaths and $88.9 \%$ of all DALYs in 2019 . Males younger than 80 years bore a larger burden of deaths than females below age 80 , as evidenced by their higher proportion of deaths (62.7\% vs $37.2 \%)$ and DALYs (63.0\% vs $37.0 \%)$. The adjusted death rates of both genders rose significantly with age, peaking at 4462.7 (95\%UI, 3237.4-5833.8) per 100,000 population at 95 plus years, and the DALY rate peaking at 24418.9 (95\%UI, 31914.7-18051.9) at 95 plus years.

\section{Global trends in NCDs attributable to dietary risks by super regions and locations}


Dietary risk-related death and DALY rates from NCDs have increased over the past 28 years in most of the GBD study super regions, except in the high-income areas, Central Europe, and the Caribbean region, which showed a decrease in 1990-2010 (Tables 1 and 2). The top three age-standardized mortality rates in the super regions were located in Eastern Europe, Oceania, and Central Asia, while the highest agestandardized DALY rates were located in Oceania, followed by Eastern Europe and Central Asia. The lowest mortality and DALY rates were observed in the high-income Asia Pacific region during 1990-2019. The disease burden was greater for men in most GBD super regions, compared to females, except in Oceania, Eastern Sub-Saharan Africa, and Western Sub-Saharan Africa. 
Table 1

Age-standardized death rates (per 100,000 population) from NCDs attributable to dietary risks by GBD super regions in 1990, 2010, and 2019.

\begin{tabular}{|c|c|c|c|c|c|c|}
\hline \multirow[t]{2}{*}{ GBD 2017 Super Region } & \multicolumn{2}{|l|}{1990} & \multicolumn{2}{|l|}{2010} & \multicolumn{2}{|l|}{2017} \\
\hline & Male & Female & Male & Female & Male & Female \\
\hline & Mean & Mean & Mean & Mean & Mean & Mean \\
\hline \multicolumn{7}{|l|}{ High-income } \\
\hline High-income Asia Pacific & 28.41 & 22.12 & 23.72 & 11.80 & 23.21 & 10.64 \\
\hline Western Europe & 72.09 & 47.52 & 54.20 & 34.11 & 50.90 & 31.85 \\
\hline Australasia & 70.72 & 52.83 & 53.98 & 40.44 & 51.13 & 38.25 \\
\hline High-income North America & 84.45 & 66.34 & 82.29 & 58.36 & 79.40 & 58.37 \\
\hline Southern Latin America & 75.05 & 53.76 & 87.81 & 58.11 & 82.34 & 56.25 \\
\hline \multicolumn{7}{|l|}{ Eastern Europe/Central Asia } \\
\hline Central Europe & 128.45 & 108.08 & 120.59 & 87.40 & 116.43 & 77.73 \\
\hline Eastern Europe & 122.59 & 105.59 & 185.27 & 122.09 & 176.52 & 104.01 \\
\hline Central Asia & 112.35 & 107.95 & 160.78 & 135.71 & 156.03 & 126.53 \\
\hline \multicolumn{7}{|l|}{ Latin America \& Caribbean } \\
\hline Tropical Latin America & 78.59 & 72.74 & 78.80 & 72.12 & 79.27 & 69.53 \\
\hline Central Latin America & 67.90 & 81.95 & 76.67 & 81.95 & 80.32 & 81.64 \\
\hline Andean Latin America & 44.88 & 46.91 & 53.24 & 59.92 & 56.00 & 58.04 \\
\hline Caribbean & 74.48 & 85.09 & 76.71 & 77.48 & 79.14 & 80.42 \\
\hline \multicolumn{7}{|c|}{ Southeast/East Asia \& Oceania } \\
\hline Southeast Asia & 28.27 & 31.34 & 45.86 & 49.66 & 50.97 & 53.72 \\
\hline East Asia & 29.49 & 28.79 & 37.06 & 35.12 & 43.22 & 38.78 \\
\hline Oceania & 120.23 & 135.18 & 181.78 & 183.53 & 169.85 & 176.46 \\
\hline \multicolumn{7}{|l|}{ North Africa \& Middle East } \\
\hline North Africa \& Middle East & 102.58 & 126.57 & 108.81 & 112.57 & 111.28 & 105.73 \\
\hline \multicolumn{7}{|l|}{ South Asia } \\
\hline South Asia & 19.21 & 20.70 & 35.99 & 40.10 & 41.08 & 51.66 \\
\hline
\end{tabular}




\begin{tabular}{|llllllll|}
\hline GBD 2017 Super Region & 1990 & & 2010 & \multicolumn{3}{c|}{2017} & \\
\cline { 2 - 7 } & Male & Female & Male & Female & Male & Female \\
\hline Southern Sub-Saharan Africa & 64.09 & 95.84 & 109.36 & 145.11 & 103.91 & 116.52 \\
\hline Eastern Sub-Saharan Africa & 26.59 & 39.02 & 37.26 & 43.67 & 38.22 & 49.41 \\
\hline Central Sub-Saharan Africa & 52.35 & 51.27 & 51.22 & 50.90 & 50.24 & 55.91 \\
\hline Western Sub-Saharan Africa & 28.85 & 45.81 & 41.51 & 60.09 & 41.31 & 57.60 \\
\hline
\end{tabular}


Table 2

Age-standardized DALY rates (per 100,000 population) from NCDs attributable to dietary risks by GBD super regions in 1990, 2010, and 2019.

\begin{tabular}{|c|c|c|c|c|c|c|}
\hline \multirow[t]{2}{*}{ GBD 2017 Super Region } & \multicolumn{2}{|l|}{1990} & \multicolumn{2}{|l|}{2010} & \multicolumn{2}{|l|}{2017} \\
\hline & Male & Female & Male & Female & Male & Female \\
\hline \multicolumn{7}{|l|}{ High-income } \\
\hline High-income Asia Pacific & 871.31 & 645.02 & 800.84 & 429.71 & 739.53 & 417.76 \\
\hline Western Europe & 1909.00 & 1190.72 & 1502.04 & 1012.49 & 1418.04 & 995.91 \\
\hline Australasia & 1922.86 & 1445.87 & 1562.10 & 1244.55 & 1557.16 & 1234.07 \\
\hline High-income North America & 2476.10 & 1940.72 & 2573.01 & 1928.34 & 2608.61 & 1934.05 \\
\hline Southern Latin America & 2144.09 & 1475.48 & 2406.27 & 1725.96 & 2295.52 & 1695.87 \\
\hline \multicolumn{7}{|l|}{ Eastern Europe/Central Asia } \\
\hline Central Europe & 3544.25 & 2688.78 & 3259.03 & 2220.21 & 2956.59 & 2044.57 \\
\hline Eastern Europe & 3298.36 & 2665.21 & 4752.83 & 3012.38 & 4079.69 & 2615.02 \\
\hline Central Asia & 3331.24 & 2902.48 & 4388.96 & 3506.48 & 4389.45 & 3323.16 \\
\hline \multicolumn{7}{|l|}{ Latin America \& Caribbean } \\
\hline Tropical Latin America & 2392.87 & 2135.43 & 2400.90 & 2105.44 & 2363.55 & 2020.70 \\
\hline Central Latin America & 2149.59 & 2453.11 & 2532.96 & 2464.81 & 2721.79 & 2491.06 \\
\hline Andean Latin America & 1426.23 & 1508.51 & 1790.89 & 1857.47 & 1694.08 & 1819.08 \\
\hline Caribbean & 2263.76 & 2544.95 & 2451.05 & 2464.74 & 2653.13 & 2549.48 \\
\hline \multicolumn{7}{|l|}{ Southeast/East Asia \& Oceania } \\
\hline Southeast Asia & 933.32 & 1059.30 & 1740.39 & 1735.65 & 2034.52 & 1901.45 \\
\hline East Asia & 840.38 & 798.02 & 1291.83 & 1005.84 & 1501.54 & 1106.54 \\
\hline Oceania & 4124.45 & 4409.01 & 5875.24 & 6017.02 & 5757.12 & 5802.55 \\
\hline \multicolumn{7}{|l|}{ North Africa \& Middle East } \\
\hline North Africa \& Middle East & 2996.19 & 3669.89 & 3285.44 & 3344.26 & 3295.64 & 3203.39 \\
\hline \multicolumn{7}{|l|}{ South Asia } \\
\hline South Asia & 573.88 & 639.54 & 1293.53 & 1242.45 & 1616.75 & 1596.83 \\
\hline \multicolumn{7}{|l|}{ Sub-Saharan Africa } \\
\hline Southern Sub-Saharan Africa & 2108.25 & 2923.45 & 3117.55 & 3881.55 & 2813.43 & 3229.96 \\
\hline
\end{tabular}




\begin{tabular}{|lllllll|}
\hline GBD 2017 Super Region & 1990 & & 2010 & \multicolumn{3}{c|}{2017} \\
\cline { 2 - 7 } & Male & Female & Male & Female & Male & Female \\
\hline Eastern Sub-Saharan Africa & 796.09 & 1074.63 & 1149.40 & 1282.09 & 1202.59 & 1470.09 \\
\hline Central Sub-Saharan Africa & 1551.75 & 1455.85 & 1543.98 & 1552.37 & 1789.40 & 1755.65 \\
\hline Western Sub-Saharan Africa & 903.03 & 1327.75 & 1311.74 & 1787.66 & 1341.35 & 1720.15 \\
\hline
\end{tabular}

The distribution of deaths and DALYs from NCDs, attributable to dietary risks by countries in 2019, are described in the global heat map (Fig. 3). The highest numbers of deaths and DALYs in the most populous nations were observed in China, with 2.0 million (1.5-2.6) deaths and 46.8 million (35.6 to 60.0) DALYs, followed by India (1.2 million deaths and 32.4 million DALYs), and the Russian Federation (0.4 million deaths and 8.5 million DALYs). The top three age-standardized death rates were found in Uzbekistan (483.6), the Solomon Islands (369.7), and Tajikistan (325.8). The top three countries with the highest age-standardized DALY rates were the Solomon Islands (9795.9), Uzbekistan (8378.9), and Nauru (7715.1). The countries with the lowest death and DALY rates were Japan (31.7) and Israel (778.9).

\section{Global trends in NCDs attributable to dietary risks by SDI}

The age-standardized rates of dietary-related deaths were highest in high-middle SDI regions, with an agestandardized rate of 176.44 (95\%UI, 162.6-188.8), while the highest DALY rate was observed in lowmiddle SDI regions with a DALY rate of $2794.2(95 \% \mathrm{UI}, 2310.0-3400.2)$ in 2019. Both rates were lowest in high SDI countries, with a death rate of $56.5(95 \% \mathrm{UI}, 46.6-68.9)$ and a DALY rate of $1308.1(95 \% \mathrm{UI}$, 1113.8-1547.0) in 2019.

Linear regression showed that the age-standardized mortality of both genders combined was negatively associated with SDI $(r=-0.2598, P<0.001)$. The mortality was $Y=-101.0 * X+185.4$. Similarly, the DALYS were negatively correlated with the SDI $(r=-0.3071, P<0.001)$, and the DALY rate was $Y=-2626 * X+4305$. Gender differences were calculated as the difference in the values of the males minus values of the females. Male gender was positively correlated with mortality $(r=0.1446, P=0.039, Y=30.39 * X+24.58)$ and a similar trend in the DALY rate $(r=0.1455, P=0.042, Y=801.0 * X+661.2)$ was found (Fig. 4A-B). The same results were found when we calculated the gender ratio as the males' rate divided by the females' rate, and the gender ratio was positively correlated with mortality $(r=0.5761, P<0.0001, \mathrm{Y}=0.9911 \star \mathrm{X}+$ $0.8555)$, showing a similar trend in the DALY rate $\left(r=0.5844,<0.001, Y=1.143^{*} X+0.8983\right)$ (Fig. 4C-D).

\section{Leading causes of deaths and DALYs from NCDs attributable to dietary risks}

Of the 23 NCDs attributable to dietary risks in the GBD data system, we analyzed the top 15 causes (Fig. 5). The top 10 causes of deaths or DALYs worldwide by NCDs attributable to dietary risks in 2019 were ischemic heart disease, stroke, diabetes mellitus, colon and rectal cancer, hypertensive heart disease, chronic kidney disease, tracheal, bronchial, and lung cancer, esophageal cancer, stomach cancer, and 
breast cancer. The mortality of ischemic heart disease ranked first in both males $(78.7,64.1-92.1)$ and females $(48.3,39.0-58.0)$. It was followed by stroke and colon and rectal cancer among males, and stroke and diabetes mellitus among females. Males and females shared the top three causes of DALY rates: ischemic heart disease, stroke, and diabetes mellitus. The rankings of five diseases on the numbers of deaths, including diabetes mellitus, tracheal, bronchial and lung cancer, chronic kidney disease, breast cancer, and atrial fibrillation and flutter rose by $72.3 \%, 39.0 \%, 72.2 \%, 37.5$, and $69.9 \%$, respectively, from 1990 to 2010 , and by $31.6 \%, 14.7 \%, 27.9 \%, 20.4 \%$, and $38.6 \%$, respectively, from 2010 to 2019 . However, the rankings on the numbers of deaths of colon and rectal cancer, stomach cancer, esophageal cancer, rheumatic heart disease, and cardiomyopathy and myocarditis declined in 1990-2019, and deaths from rheumatic heart disease showed a significant downward trend after 1990 (Fig. 5A).

The five leading causes of death from ischemic heart disease, stroke, colon and rectal cancer, diabetes mellitus, and hypertensive heart disease increased by $27.6 \%, 16.2 \%, 63.5 \%, 72.3 \%$, and $3.5 \%$, respectively, during 1990-2010. In 2010-2019, the five leading causes of death from ischemic heart disease, stroke, diabetes mellitus, colon and rectal cancer, and hypertensive heart disease increased by $16.9 \%, 8.4 \%$, $31.6 \%, 24.6 \%$, and $31.2 \%$, respectively (Fig. $5 \mathrm{~A}$ ). The ranking of diabetes rose from 4 th to $3 \mathrm{rd}$. A similar trend was found in the numbers of DALYs, with an $85.2 \%$ and $34.3 \%$ increase during the two periods, respectively. The top five causes of DALYs remained stable in the two periods (Fig. 5B).

\section{Leading risks of deaths and DALYs from NCDs attributable to dietary risks}

The top five dietary risks for death or DALYs attributable to NCDs remained stable during the two periods. These risks during 1990-2019 included diets high in sodium, low in whole grains, low in legumes, low in fruits, and high in red meat. Diets high in trans fats increased significantly during the two periods by $17.9 \%$ and $18.3 \%$ respectively, from a ranking of 8 th to 6 th. The other three diet risks, including diets low in nuts and seeds, low in polyunsaturated fatty acids, and low in omega-3 fatty acids moved up one place during the past 29 years. The rankings of diets low in nuts and seeds moved from 9th to 8th, and the rankings of diets high in trans fats remained at 8th in 1990-2019, as measured by the DALYs (Fig. 6B). However, diets low in vegetables and high in processed meat, showed large declines in rankings (Fig. 6A), and diets low in vegetables declined by $39.5 \%$, and the DALY rate declined by $11.0 \%$ (Fig. 6B).

\section{Discussion}

This study's results support reports of trends in deaths and DALYs from NCDs attributable to dietary risks by age, sex, and region in 204 countries from 1990 to 2019. Dietary risk-related NCDs accounted for an increase in all-cause deaths from 18.9-22.7\%, and all-cause DALYs from 9.9-15.5\%, over the past 29 years. We concluded that 7.9 million deaths and 187.7 million DALYs from NCDs worldwide in 2019 were related to dietary risks that increased $146.7 \%$, compared to the 1990 increase of $142.1 \%$. Both mortality and DALY rates decreased significantly, after age was standardized to adjust for population and age structure, indicating an overall increase in the numbers of deaths and DALYs related to population 
growth and the aging population. During the same period, the proportion of dietary risk-related deaths and DALYs remained stable; these trends might have been driven by decreases in background mortality. In 2019 , dietary risk-related cardiovascular disease accounted for $85.5 \%$ of deaths and $80.8 \%$ of DALYs; dietary risk-related cancers accounted for $7.6 \%$ of deaths and $7.4 \%$ of DALYs; and dietary risk-related diabetes accounted for $4.8 \%$ of deaths and $9.6 \%$ of DALYs.

Given the overall changes in the DALYs and deaths from dietary risks-related NCDs, their rates are controversial. We suspect the increase in the numbers of deaths and DALYs might have resulted from population growth and an aging population.

The analysis by age revealed a single-peak distribution of numbers of deaths or DALYs of both genders. The all-age adjusted mortality and DALY rates increased significantly with age, supporting age as the risk factor with the greatest impact. Gender disparities have fluctuated over the past 29 years. Males had higher numbers of deaths and DALYs than females at a relatively young age, and females exceeded those numbers at age 80-84 years. After the data were adjusted, no gender differences were found in the overall rates across all ages. We speculate that the intersection might have been related to a significantly higher life expectancy of females.

The analysis by location showed that countries with the highest numbers of deaths or DALYs were China, India, the Russian Federation, the United States, and Indonesia, which have larger populations. After the data were standardized by population and age structure, the countries with the highest death rates and DALYs were Uzbekistan and the Solomon Islands, respectively. The burden of dietary risk-related NCDs was negatively associated with the SDI. Except for the high SDI regions, all regions experienced significant increases in the numbers of deaths and DALYs. People in higher socioeconomic groups were more likely to have healthier diets, characterized by a high intake of fruits and vegetables and low in intake of trans fats and processed foods ${ }^{6}$. People in low- and middle-income countries had a large increase in NCDs and their risk factors, which were considered outcomes of urbanization, nutritional transition (from a traditional diet to a classical western diet), and an aging population ${ }^{7,8}$. However, people in high-SDI regions consumed more fat, salt, and processed foods ${ }^{9}$. Thus, many factors have contributed to sharp increases in the dietary risk-related disease burden worldwide, which might not simply be associated with socioeconomic level, but largely with personal determining factors.

Dietary habits depend on total monthly income, with low incomes more likely to result in poor eating and feeding habits and food insecurity. Low SDI regions have less access to healthy foods and high-quality medical care because of the high costs of fresh food and medical services, which are barriers to early education, screening, diagnosis, and treatment of NCDs. High SDI regions have more access to fresh fruits and vegetables and medical education, increasing awareness of disease prevention and healthy lifestyles.

Gender-specific disparities were found at several levels in this study. Males of different ages living in different SDI regions suffered more than females, as measured by deaths and DALYs from 1990 to 2019 . 
These differences might be related to the higher mortality and DALY rates of cardiovascular disease and cancer among males under age $70^{10,11}$. The age of the males with the highest number of deaths or DALYs was much younger than age of the females, which may be attributed to the protective effects of estrogen before menopause. Estrogen has antioxidant and antiapoptotic effects on cardiomyocytes in ischemia ${ }^{12}$. The high burden of NCDs in males may be attributed to the less attention paid to body mass index (BMI), as high-caloric food intake and high consumption of alcohol are more likely found in males. This finding could have been partially due to the greater exposure of males to potentially aggravating factors (e.g., less physical activity, more smoking, and work-related stress).

Differences in deaths or DALYs rates by gender difference and ratio were positively associated with the SDI. However, in some relatively low-SDI regions females had a greater burden of NCDs attributed to dietary risks, consistent with previous studies ${ }^{13,14}$. In this study, the death and DALY rates of females exceeded those of males in a few low SDI regions. The reasons may be related to less education and medical-care access and high BMI in low-SDI countries ${ }^{15}$. Therefore, target measures are crucial for males and females from different socioeconomic contexts. The leading causes of deaths or DALYs from NCDs attributable to dietary risks, included ischemic heart disease, stroke, diabetes mellitus, stomach cancer, colon and rectal cancer, and hypertensive heart disease, related to diets high in sodium sugar ${ }^{15-}$ 19, saturated fats, trans fats, and low in omega-3 fatty acids and fiber [14-18]. Diabetes-related deaths and mortality have increased over the past 29 years, as evidenced by DALYs. The global health and financial burdens of diabetes have increased, and are expected to grow in the coming years, which indicates that diabetes is a public health threat, worldwide. Implementation of cost-effective treatments, early diagnosis, and prevention strategies for diabetes are strongly encouraged. Nevertheless, the DALY and mortality rates of rheumatic heart disease have declined, which is likely related to the high cure rate of rheumatism from the wide use of penicillin. However, the prevalence of dietary-related gastrointestinal cancer remains high, with a significant decline in the death and DALY rates. Another interesting phenomenon is the increase in the mortality and DALY rates of atrial fibrillation and flutter related to dietary risks since 1990; however, a PubMed search on this topic did not reveal a potential research direction.

Although sodium, sugar, and fat have been thought of as killers in public health, our analysis shows that the leading dietary risk factors for mortality and DALYs are diets high in sodium and low in whole grains, fruits, legumes, fiber, vegetables, and omega- 3 fatty acids, each one causing more than $2 \%$ of global deaths. We found that people in regions with high numbers of deaths and DALYs from NCDs (e.g., China) are more likely to have high-sodium intake. Dietary risk-related NCDs attributable to diets low in milk and calcium consumption are associated with rising DALYs. Hence, promoting and improving the intake of some dietary nutrients (i.e., calcium) and reducing the intake of others (i.e., sodium and fat) deserve attention.

Herein, we conducted a comprehensive analysis of data from the GBD website on dietary risk-related NCDs during 1990-2019. To our knowledge, this study offers the most comprehensive, accurate, and up- 
to-date information about the burdens of dietary risks by year, age, sex, SDI level, and region. It also provides an overview of the leading causes of NCDs attributable to dietary risks.

Our study has limitations. First, as with other analyses of disease burden, our study has the same methodological limitations as the 2019 GBD study. Second, dietary risks might be just one of several high risks for NCDs, as the GBD data has unavoidable confounding factors. We could not eliminate the confounding factors in dietary risk-related NCDs from other causes.

\section{Conclusion}

This study's evaluation of the burdens of NCDs attributable to dietary risks showed a continuous global trend in the growth of dietary risk-related NCDs. The mortality and DALY rates showed a decreasing trend by year. However, age distribution and calendar year showed substantial differences between the male and female groups, with males in countries having lower SDIs showing a greater disease burden due to dietary risks. Thus, we recommend the implementation of cost-effective policies that promote optimal dietary intake as a preventive measure to avoid the occurrence of NCDs.

\section{List Of Abbreviations}

DALY, disability-adjusted life-years; GBD, Global Burden of Disease; NCD, Non-communicable disease; SDI, Socio-demographic Index

\section{Declarations}

Ethics approval and consent to participate: Not applicable

Consent for publication: Not applicable

Availability of data and materials: The datasets generated and/or analysed during the current study are available in the http://www.healthdata.org/gbd/20196.

Competing interests: The authors declare that they have no competing interests.

Funding: The support funding from National Natural Science Foundation of China.

Authors' contributions: Jie Qiao analyzed and interpreted all of data from the dataset, and was a major contributor in writing the manuscript. Xiling Lin complet graphics drawing. Yiwen Wu, Xin Huang' Xiaowen Pan, Jingya Xu, JunYun Wu download and sort the data from the website All authors read and approved the final manuscript.

Acknowledgements: Not applicable

\section{References}


1. GBD 2017 Diet Collaborators. Health effects of dietary risks in 195 countries, 1990-2017: a systematic analysis for the Global Burden of Disease Study 2017. Lancet. 2019 May 11;393(10184):1958-1972. doi: 10.1016/S0140-6736(19)30041-8. Epub 2019 Apr 4. PMID: $30954305 ;$ PMCID: PMC6899507.

2. GBD 2017 DALYs and HALE Collaborators. Global, regional, and national disability-adjusted life-years (DALYs) for 359 diseases and injuries and healthy life expectancy (HALE) for 195 countries and territories, 1990-2017: a systematic analysis for the Global Burden of Disease Study 2017. Lancet. 2018 Nov 10;392(10159):1859-1922. doi: 10.1016/S0140-6736(18)32335-3. Erratum in: Lancet. 2019 Jun 22;393(10190):e44. PMID: 30415748; PMCID: PMC6252083.

3. GBD 2017 Causes of Death Collaborators. Global, regional, and national age-sex-specific mortality for 282 causes of death in 195 countries and territories, 1980-2017: a systematic analysis for the Global Burden of Disease Study 2017. Lancet. 2018 Nov 10;392(10159):1736-1788. doi: 10.1016/S0140-6736(18)32203-7. Epub 2018 Nov 8. Erratum in: Lancet. 2019 Jun 22;393(10190):e44. Erratum in: Lancet. 2018 Nov 17;392(10160):2170. PMID: 30496103; PMCID: PMC6227606.

4. Ezzati M, Pearson-Stuttard J, Bennett JE, Mathers CD. Acting on non-communicable diseases in lowand middle-income tropical countries. Nature. 2018.

5. The World Health Organization's Global Action Plan for the Prevention of NCDs 2013-2020 https://www.who.int/nmh/publications/ncd-action-plan/en/ .

6. Global Burden of Disease (GBD) Study http://www.healthdata.org/gbd/2019.

7. Marcone MF, Madan P, Grodzinski B. An Overview of the Sociological and Environmental Factors Influencing Eating Food Behavior in Canada. Front Nutr. 2020.

8. Murphy GA, et al. Sociodemographic distribution of non-communicable disease risk factors in rural Uganda: a cross-sectional study. Int J Epidemiol. 2016.

9. Allen L, Williams J, Townsend N, et al. Socioeconomic status and non-communicable disease behavioural risk factors in low-income and lower-middle-income countries: a systematic review. Lancet Glob Health. 2017.

10. Giskes K, Avendano M, Brug J, Kunst AE. A systematic review of studies on socioeconomic inequalities in dietary intakes associated with weight gain and overweight/obesity conducted among European adults. Obes Rev. 2010.

11. Alabas OA, Gale CP, Hall M, et al. Sex Differences in Treatments, Relative Survival, and Excess Mortality Following Acute Myocardial Infarction: National Cohort Study Using the SWEDEHEART Registry. J Am Heart Assoc. 2017. .

12. Kim HI, Lim H, Moon A. Sex Differences in Cancer: Epidemiology, Genetics and Therapy. Biomol Ther (Seoul). 2018 Jul 1;26(4):335-342. doi: 10.4062/biomolther.2018.103. PMID: 29949843; PMCID: PMC6029678.

13. Morselli E, Santos RS, Criollo A, Nelson MD, Palmer BF, Clegg DJ. The effects of oestrogens and their receptors on cardiometabolic health. Nat Rev Endocrinol. 2017. 
14. Song QB, Zhao Y, Liu YQ, Zhang J, Xin SJ, Dong GH. Sex difference in the prevalence of metabolic syndrome and cardiovascular-related risk factors in urban adults from 33 communities of China: The CHPSNE study. Diab Vasc Dis Res. 2015. .

15. Dong GH, Wang D, Liu MM, et al. Sex difference of the prevalence and risk factors associated with prehypertension among urban Chinese adults from 33 communities of China: the CHPSNE study. J Hypertens. 2012. .

16. Calu Costa J, Wehrmeister FC, Barros AJ, Victora CG. Gender bias in careseeking practices in 57 lowand middle-income countries. J Glob Health. 2017. .

17. Moorley C, Cahill S, Corcoran N. Stroke among African-Caribbean women: lay beliefs of risks and causes. J Clin Nurs. 2016.

18. Sieri S, Agnoli C, Pala V, et al. Dietary glycemic index, glycemic load, and cancer risk: results from the EPIC-Italy study. Sci Rep. 2017. .

19. Hidayat K, Yang CM, Shi BM. Body fatness at an early age and risk of colorectal cancer. Int J Cancer. 2018 .

20. GBD 2017 Stomach Cancer Collaborators. The global, regional, and national burden of stomach cancer in 195 countries, 1990-2017: a systematic analysis for the Global Burden of Disease study 2017. Lancet Gastroenterol Hepatol. 2020 Jan;5(1):42-54. doi: 10.1016/S2468-1253(19)30328-0. Epub 2019 Oct 21. Erratum in: Lancet Gastroenterol Hepatol. 2020 Mar;5(3):e2. PMID: 31648970; PMCID: PMC7033564.

21. Haidong W, Kaja M .A, Mitra A, GBD 2019 Demographics Collaborators. Global age-sex-specific fertility, mortality, healthy life expectancy (HALE), and population estimates in 204 countries and territories, 1950-2019: a comprehensive demographic analysis for the Global Burden of Disease Study 2019. Lancet. 2020 Oct 17;396(10258):1160-1203. doi: 10.1016/S0140-6736(20)30977-6. PMID: 33069325; PMCID: PMC7566045.

\section{Figures}


A

Global Death Number of NCDs attribute to Dietary Risks

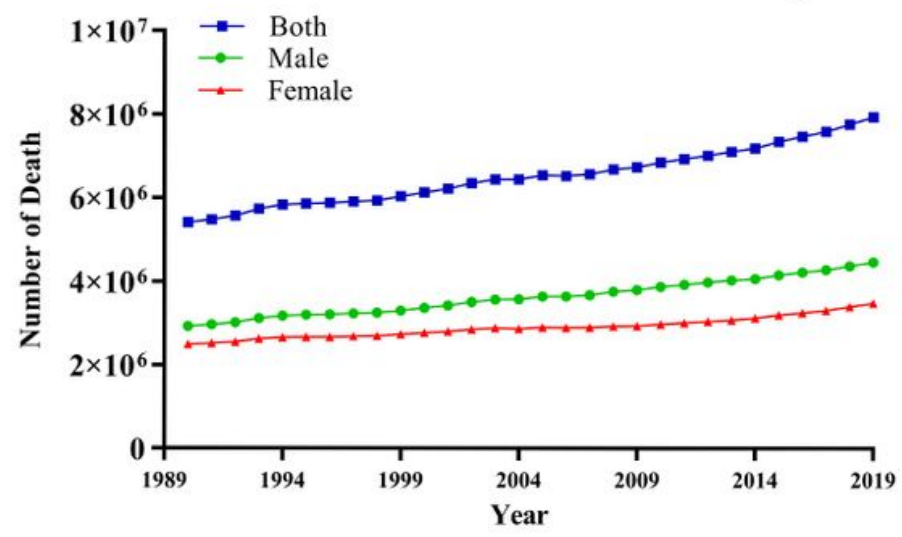

C

Global DALYs Number of NCDS attribute to Dietary Risks

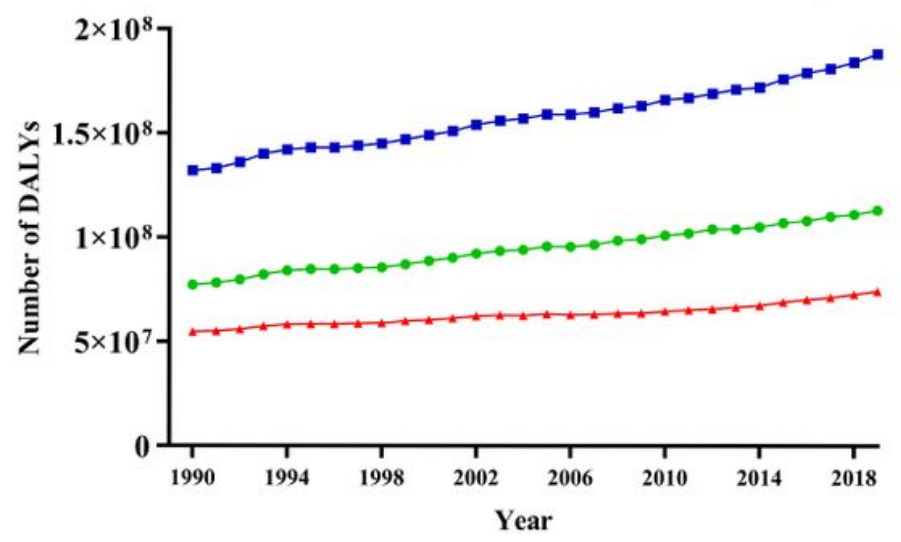

B Global Mortaliity of NCDs attribute to Dietary Risks

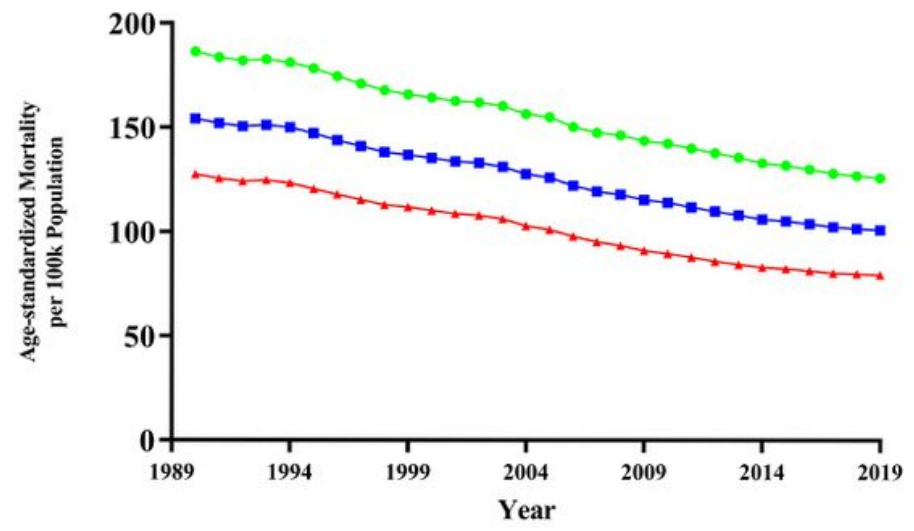

D

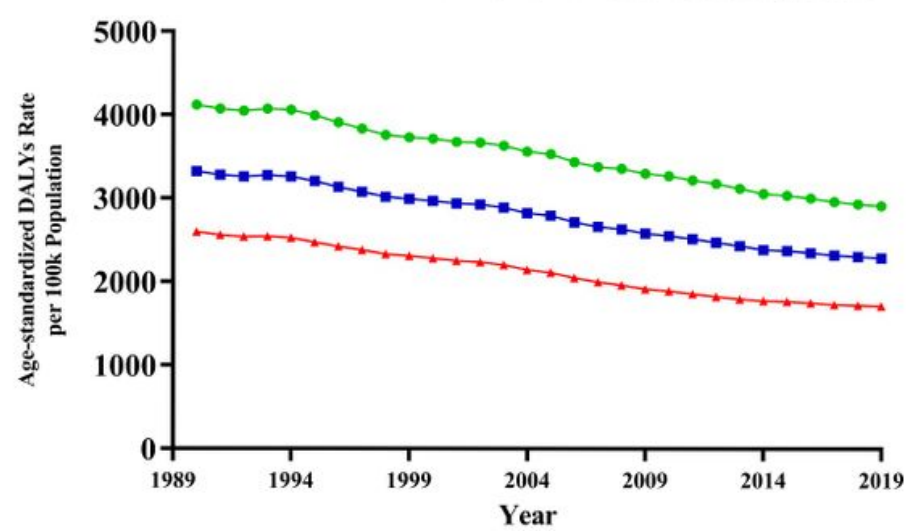

\section{Figure 1}

Global burden of NCDs attributable to dietary risks from 1990 to 2019.

Figure 2

Global burden of NCDs attributable to dietary risks by age in 2019. 
A

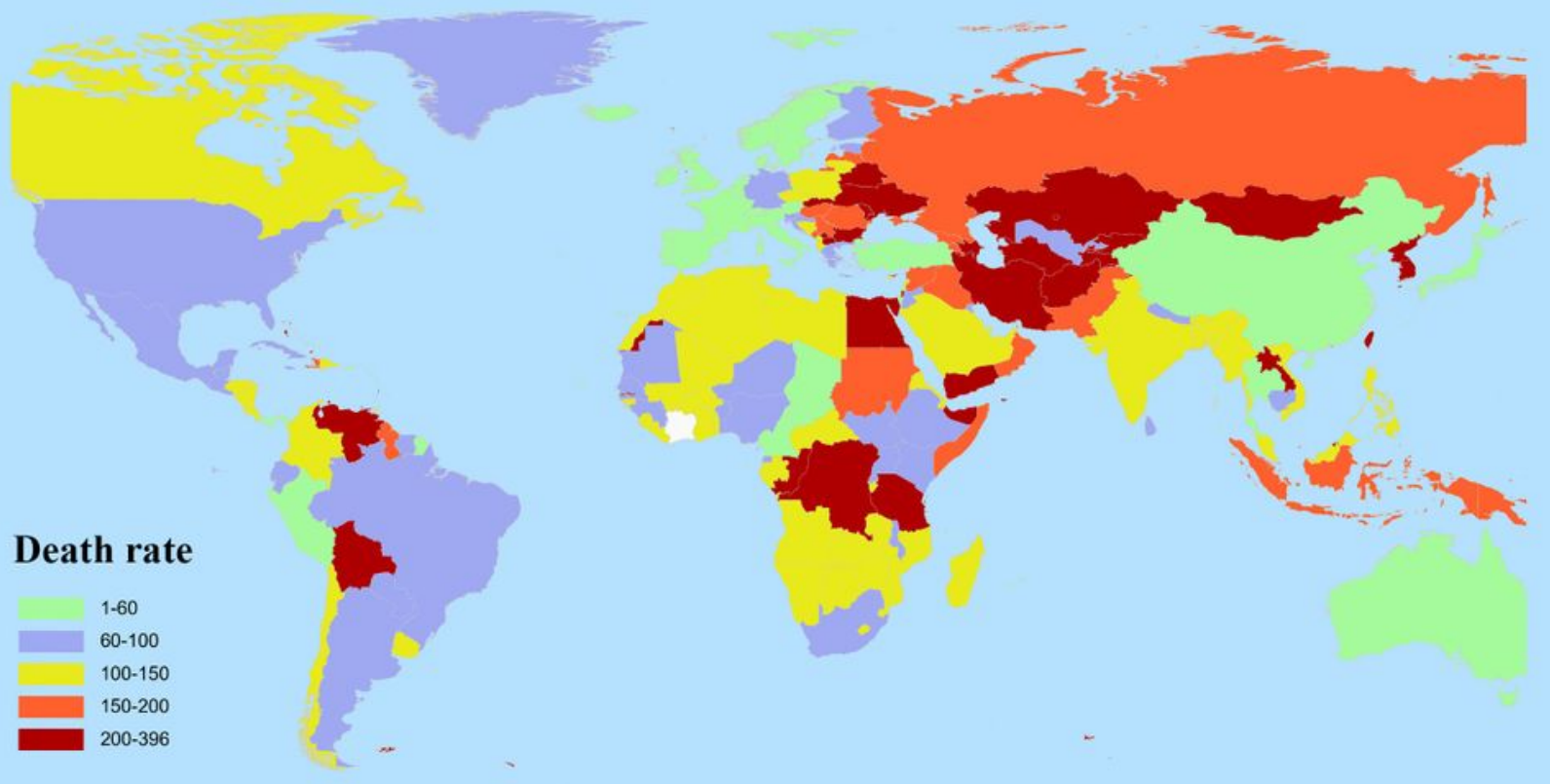

B

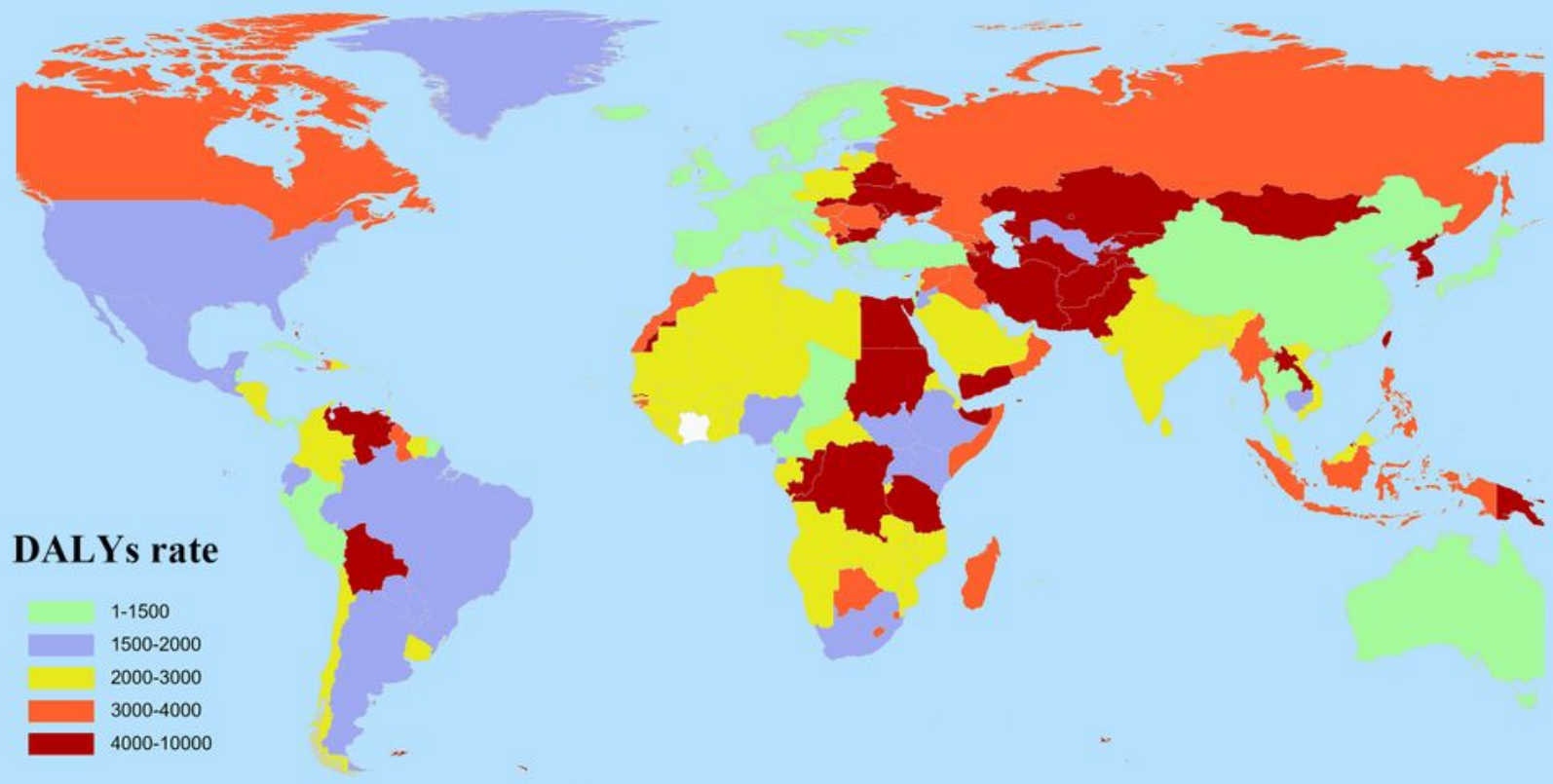

Figure 3

AB. Global map of the NCD burden attributable to dietary risks in 2019. Note: The designations employed and the presentation of the material on this map do not imply the expression of any opinion whatsoever on the part of Research Square concerning the legal status of any country, territory, city or area or of its authorities, or concerning the delimitation of its frontiers or boundaries. This map has been provided by the authors. 


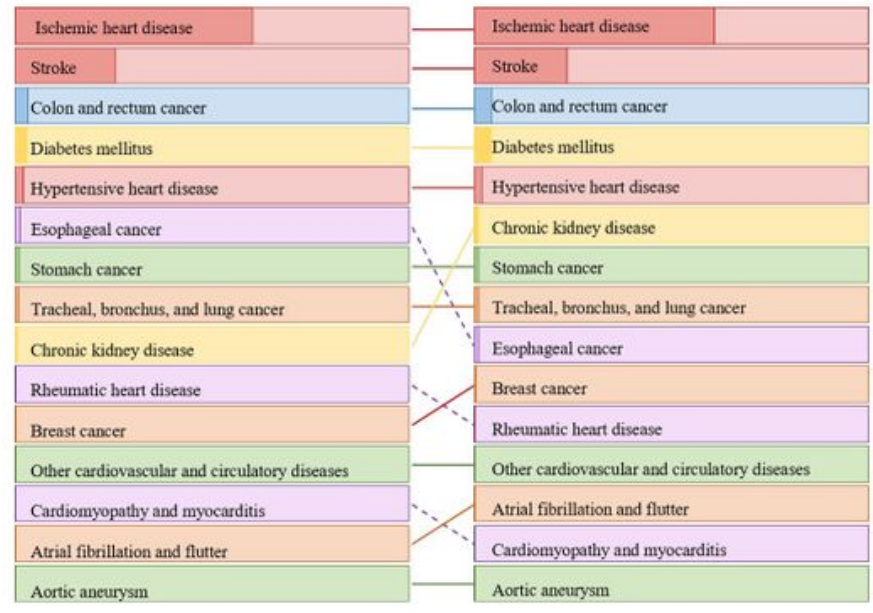

\begin{tabular}{|l|}
\hline $27.6 \%$ \\
\hline $16.2 \%$ \\
\hline $63.5 \%$ \\
\hline $72.3 \%$ \\
\hline $3.5 \%$ \\
\hline $72.2 \%$ \\
\hline $18.7 \%$ \\
\hline $39.0 \%$ \\
\hline$-7.7 \%$ \\
\hline $37.5 \%$ \\
\hline$-31.8 \%$ \\
\hline $4.4 \%$ \\
\hline $09.9 \%$ \\
\hline $18.3 \%$ \\
\hline $48.6 \%$ \\
\hline
\end{tabular}

\begin{tabular}{|l|l|l|}
\hline$-26.7 \%$ & & \\
\hline$-30.8 \%$ & Ischemic heart disease \\
\hline $2.6 \%$ & Stroke \\
\hline$-2.7 \%$ & Diabetes mellitus \\
\hline$-37.2 \%$ & & \\
\hline $5.0 \%$ & Colon and rectum cancer \\
\hline$-26.8 \%$ & Hypertensive heart disease \\
\hline$-13.2 \%$ & Chronic kidney disease \\
\hline$-42.9 \%$ & Tracheal, bronchus, and lung cancer \\
\hline$-16.2 \%$ & Stomach cancer \\
\hline$-56.9 \%$ & Esophageal cancer \\
\hline$-5.0 \%$ & Breast cancer \\
\hline$-37.1 \%$ & Atrial fibrillation and flutter \\
\hline$-32.9 \%$ & \\
\hline$-9.2 \%$ & Other cardiovascular and circulatory diseases \\
\hline & Rheumatic heart disease \\
\hline
\end{tabular}

\begin{tabular}{|l|l|}
\hline $16.9 \%$ & $-11.0 \%$ \\
\hline $8.4 \%$ & $-16.6 \%$ \\
\hline $31.6 \%$ & $0.1 \%$ \\
\hline $24.6 \%$ & $-4.5 \%$ \\
\hline $31.2 \%$ & $-0.4 \%$ \\
\hline $27.9 \%$ & $-2.2 \%$ \\
\hline $14.7 \%$ & $-11.3 \%$ \\
\hline $2.4 \%$ & $-20.3 \%$ \\
\hline $2.4 \%$ & $-20.6 \%$ \\
\hline $20.4 \%$ & $-5.8 \%$ \\
\hline $38.6 \%$ & $2.0 \%$ \\
\hline $16.9 \%$ & $-9.3 \%$ \\
\hline$-8.7 \%$ & $-27.7 \%$ \\
\hline $18.9 \%$ & $-8.7 \%$ \\
\hline $25.2 \%$ & $-3.6 \%$ \\
\hline
\end{tabular}

B

\begin{tabular}{|l|l|l|}
\hline Ischemic heart disease \\
\hline Stroke & Ischemic heart disease \\
\hline Diabetes mellitus \\
\hline Colon and rectum cancer \\
\hline Hypertensive heart disease \\
\hline Esophageal cancer \\
\hline Stomach cancer \\
\hline Chronic kidney disease \\
\hline Tracheal, bronchus, and lung cancer \\
\hline Rheumatic heart disease \\
\hline Breast cancer \\
\hline \hline Other cardiovascular and circulatory diseases \\
\hline Atrial fibrillation and flutter \\
\hline Cardiomyopathy and myocarditis \\
\hline Aortic aneurysm & Stomach cancer \\
\hline
\end{tabular}

\begin{tabular}{|l|}
\hline $25.4 \%$ \\
\hline $13.6 \%$ \\
\hline $85.2 \%$ \\
\hline $57.5 \%$ \\
\hline$-3.1 \%$ \\
\hline $62.1 \%$ \\
\hline $10.6 \%$ \\
\hline$-11.7 \%$ \\
\hline $26.7 \%$ \\
\hline $35.5 \%$ \\
\hline$-30.8 \%$ \\
\hline $56.4 \%$ \\
\hline $13.1 \%$ \\
\hline $24.9 \%$ \\
\hline $44.2 \%$ \\
\hline
\end{tabular}

\begin{tabular}{|c|c|c|}
\hline $.23 .9 \%$ & \multicolumn{2}{|c|}{ Ischemic heart disease } \\
\hline$-29.6 \%$ & Stroke & \\
\hline $14.5 \%$ & \multicolumn{2}{|c|}{ Diabetes mellitus } \\
\hline$-2.7 \%$ & \multicolumn{2}{|c|}{ Colon and rectum cancer } \\
\hline$-39.9 \%$ & \multicolumn{2}{|c|}{ Hypertensive heart disease } \\
\hline $2.0 \%$ & \multicolumn{2}{|c|}{ Chronic kidney disease } \\
\hline$-30.4 \%$ & \multicolumn{2}{|c|}{ Tracheal, bronchus, and lung cancer } \\
\hline $.44 .8 \%$ & \multicolumn{2}{|c|}{ Stomach cancer } \\
\hline$-20.0 \%$ & \multicolumn{2}{|c|}{ Esophageal cancer } \\
\hline$-15.5 \%$ & \multicolumn{2}{|c|}{ Breast cancer } \\
\hline$-5.3 \%$ & \multicolumn{2}{|c|}{ Atrial fibrillation and flutter } \\
\hline$-55.6 \%$ & \multicolumn{2}{|c|}{ Rheumatic heart disease } \\
\hline$-29.6 \%$ & \multicolumn{2}{|c|}{ Other cardiovascular and circulatory disease } \\
\hline$-24.6 \%$ & \multicolumn{2}{|c|}{ Cardiomyopathy and myocarditis } \\
\hline$-10.2 \%$ & \multicolumn{2}{|c|}{ Aortic aneurysm } \\
\hline
\end{tabular}

\begin{tabular}{|l|l|}
\hline $12.7 \%$ & $-11.0 \%$ \\
\hline $6.8 \%$ & $-15.3 \%$ \\
\hline \hline $34.3 \%$ & $5.8 \%$ \\
\hline $21.2 \%$ & $-4.3 \%$ \\
\hline $24.2 \%$ & $-3.1 \%$ \\
\hline $21.2 \%$ & $-4.2 \%$ \\
\hline $10.7 \%$ & $-12.9 \%$ \\
\hline$-1.9 \%$ & $-22.0 \%$ \\
\hline $0.2 \%$ & $-20.6 \%$ \\
\hline \hline $17.2 \%$ & $-5.1 \%$ \\
\hline $32.9 \%$ & $1.9 \%$ \\
\hline \hline$-9.5 \%$ & $-25.6 \%$ \\
\hline $13.9 \%$ & $-8.2 \%$ \\
\hline $15.8 \%$ & $-7.3 \%$ \\
\hline \hline $20.7 \%$ & $-4.9 \%$ \\
\hline
\end{tabular}

\section{Figure 4}

A-B. Leading causes of deaths and DALYs from NCDs attributable to dietary risks in 2019 


\section{Leading risks in 1990}

\section{Leading risks in 2010}

Changes in age-

in Death standardize number Death rate

\begin{tabular}{|l|l|}
\hline Diet high in sodium & Diet high in sodium \\
\hline Diet low in whole grains & Diet low in whole grains \\
\hline Diet low in legumes & Diet low in legumes \\
\hline Diet low in fruits & Diet low in fiber \\
\hline Diet high in red meat & Diet high in trans fatty acids \\
\hline Diet low in fiber & Diet low in nuts and seeds \\
\hline Diet low in vegetables & Diet low in polyunsaturated fatty acids \\
\hline Diet low in nuts and seeds & Diet low in seafood omega-3 fatty acids \\
\hline Diet high in processed meat & Diet high in processed meat \\
\hline Diet low in polyunsaturated fatty acids & Diet low in milk \\
\hline Diet low in seafood omega- 3 fatty acids & Diet low in calcium \\
\hline Diet high in sugar-sweetened beverages & \\
\hline Diet low in milk & \\
\hline \hline Diet low in calcium & \\
\hline
\end{tabular}

\begin{tabular}{|c|c|c|}
\hline $24.6 \%$ & $-25.3 \%$ & Diet high in sodium \\
\hline $33.2 \%$ & $-23.2 \%$ & Diet low in whole grains \\
\hline $20.4 \%$ & $-30.5 \%$ & Diet low in legumes \\
\hline $19.4 \%$ & $-28.6 \%$ & Diet low in fruits \\
\hline $30.8 \%$ & $-23.2 \%$ & Diet high in red meat \\
\hline $14.1 \%$ & $-34.2 \%$ & Diet high in trans fatty acids \\
\hline $17.9 \%$ & $-32.1 \%$ & Diet low in fiber \\
\hline $21.4 \%$ & $-30.1 \%$ & Diet low in nuts and seeds \\
\hline$-0.6 \%$ & $-41.3 \%$ & Diet low in vegetables \\
\hline $32.3 \%$ & $-22.5 \%$ & Diet low in polyunsaturated fatty acids \\
\hline $32.7 \%$ & $-23.5 \%$ & Diet low in seafood omega- 3 fatty acids \\
\hline $20.5 \%$ & $-30.3 \%$ & Diet high in processed meat \\
\hline $33.4 \%$ & $-22.9 \%$ & Diet high in sugar-sweetened beverages \\
\hline $74.1 \%$ & $4.0 \%$ & Diet low in milk \\
\hline $74.8 \%$ & $4.6 \%$ & Diet low in calcium \\
\hline
\end{tabular}

Changes

Changes in age-

in DALYs standardized

number DALYs rate
Changes in age-

in Death standardized

number Death rate

B

\section{Leading risks in 1990}

\section{Leading risks in 2010}

\begin{tabular}{|l|l|}
\hline Diet high in sodium \\
\hline Diet low in whole grains \\
\hline Diet low in fruits \\
\hline Diet low in legumes \\
\hline Diet low in red meat \\
\hline Diet high in trans fatty acids \\
\hline Diet low in vegetables \\
\hline Diet high in processed meat \\
\hline Diet low in polyunsaturated fatty acids \\
\hline Diet low in seafood omega- 3 fatty acids \\
\hline Diet high in sugar-sweetened beverages \\
\hline Diet low in milk \\
\hline Diet low in calcium \\
\hline
\end{tabular}

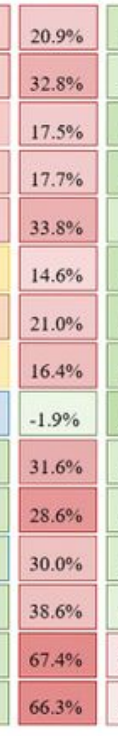

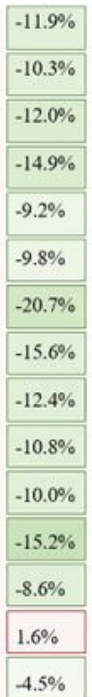

Changes

Changes in age-

Leading risks in 2019 number DALYs rate

\begin{tabular}{|l|}
\hline Diet high in sodium \\
\hline Diet low in whole grains \\
\hline Diet low in fruits \\
\hline Diet low in legumes \\
\hline Diet high in red meat \\
\hline Diet low in fiber \\
\hline Diet low in vegetables \\
\hline Diet high in trans fatty acids \\
\hline Diet low in nuts and seeds \\
\hline Diet high in processed meat \\
\hline Diet low in polyunsaturated fatty acids \\
\hline Diet low in seafood omega-3 fatty acids \\
\hline Diet high in sugar-sweetened beverages \\
\hline Diet low in milk \\
\hline Diet low in calcium \\
\hline
\end{tabular}

\begin{tabular}{|l|c|}
\hline $11.1 \%$ & $-12.4 \%$ \\
\hline $14.5 \%$ & $-9.3 \%$ \\
\hline $8.5 \%$ & $-13.3 \%$ \\
\hline $10.8 \%$ & $-12.0 \%$ \\
\hline $17.0 \%$ & $-7.0 \%$ \\
\hline $0.0 \%$ & $-19.6 \%$ \\
\hline \hline $13.8 \%$ & $-9.6 \%$ \\
\hline $7.4 \%$ & $-14.4 \%$ \\
\hline $11.4 \%$ & $-11.0 \%$ \\
\hline \hline $14.0 \%$ & $-10.3 \%$ \\
\hline $11.2 \%$ & $-11.0 \%$ \\
\hline \hline $13.0 \%$ & $-10.1 \%$ \\
\hline $23.0 \%$ & $-3.2 \%$ \\
\hline $28.7 \%$ & $1.7 \%$ \\
\hline $19.8 \%$ & $-5.0 \%$ \\
\hline
\end{tabular}

\section{Figure 5}

A-B. Leading risks of deaths and DALYs from NCDs attributable to dietary risks in 2019 
A

Gender Difference of Age-standardized Death by SDI

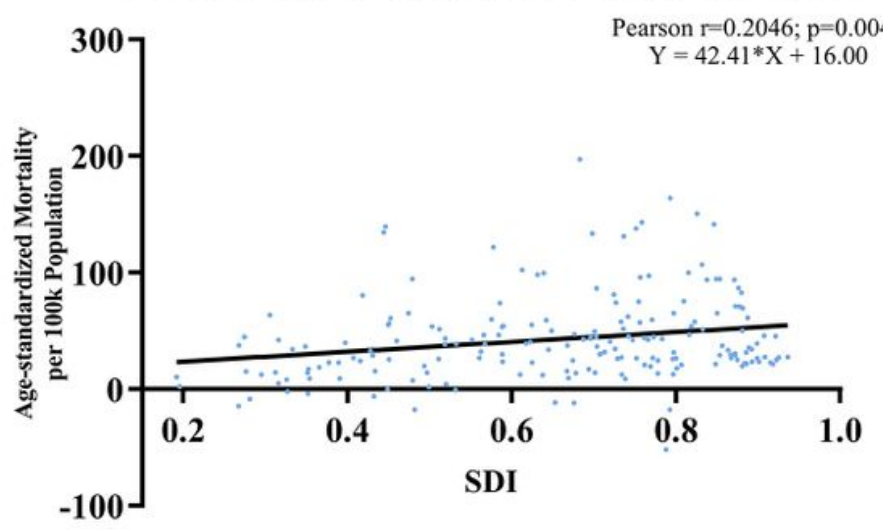

B

Gender Difference of Age-standardized DALYs by SDI

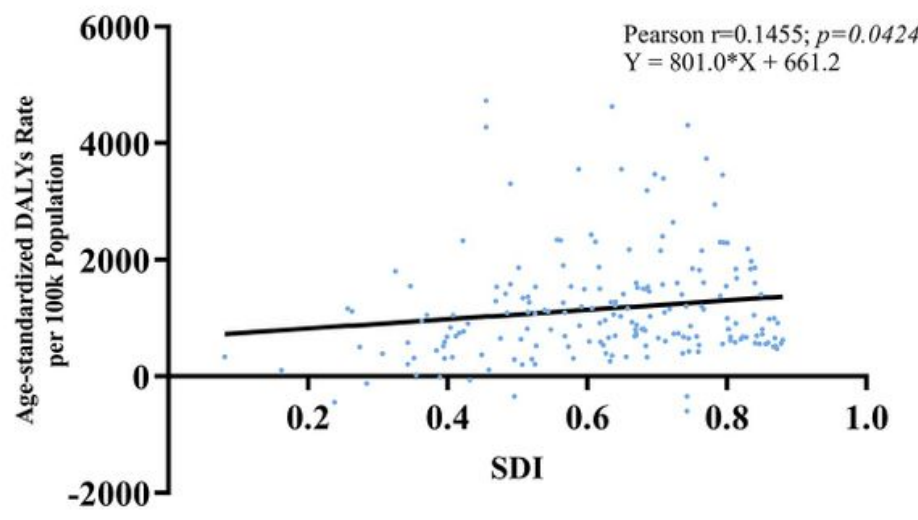

C

Gender Ratio of Age-standardized Death by SDI

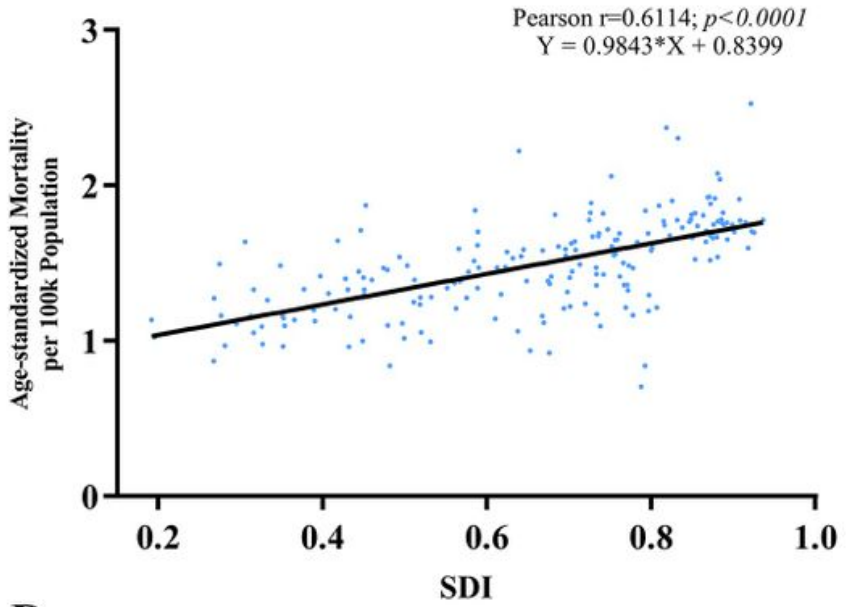

D

Gender Ratio of Age-standardized DALYs by SDI

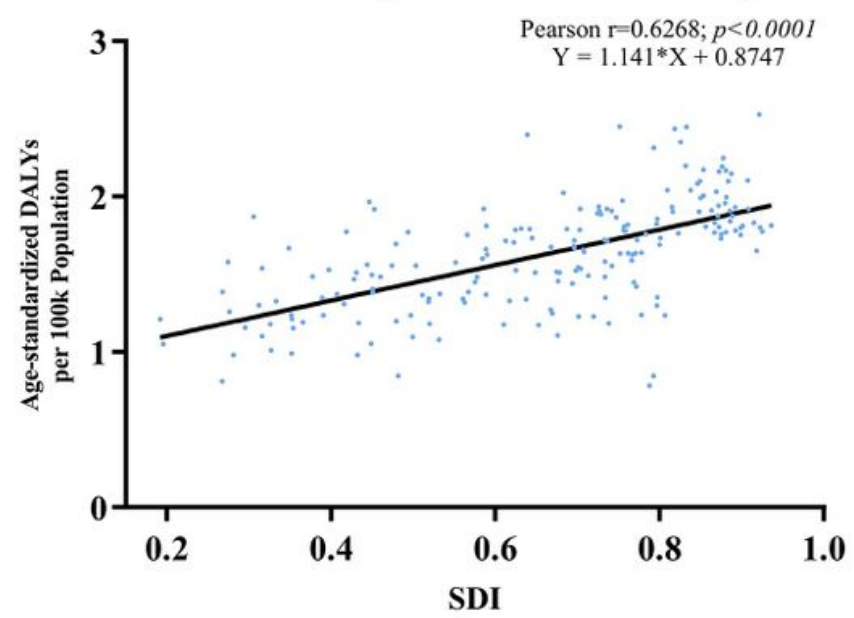

Figure 6

A-B Burden of NCDs attributable to dietary risks by SDI and gender in 2019 . 